\title{
Expectations for Presentation of Engineering and Scientific Mobile Platform Information within a Virtual Globe Geographic Information Systems
}

\author{
Brian Guise, Michael D. Proctor \\ University of Central Florida, Florida, USA \\ E-mail: brian.guise@l-3com.com,mproctor@ucf.edu \\ Received January 9, 2011; revised January 17, 2011; accepted January 20, 2011
}

\begin{abstract}
Layered information systems like Google Earth have revolutionized public access to and visualization of geographic information through virtual globes. Separately, geo-specific technical information has been advanced in mobile platforms, both handheld and embedded devices, for the engineering and scientific communities. However, engineering and scientific information has had limited penetration into virtual globe Geographic Information Systems (GIS). This article explores unmet expectations which may be at the root of the issue. These expectations include design of the architecture within the originating mobile platform as well as expression of the level of accuracy and precision necessary for validity of the simulation displayed through the virtual globe GIS. The article below discusses architecture and validity research that advances real-time generation of simulated electro-magnetic coverage maps as composed layers within a mobile platform. Further, the research also enables real-time visualization of the simulated coverage maps by a global team through a virtual globe. Finally, for communication assurance purposes, the level of validity of the generated simulated coverage maps are analyzed from the perspective of an analog celestial body exploration mission by a mobile rover and its supporting organization analysis needs.
\end{abstract}

Keywords: Communication Simulation, Layered Architecture, Validity

\section{Introduction}

Geo-specific terrain information such as satellite imagery, aerial photography, and digital map data within the context of Geographic Information Systems (GIS) is commonly represented as layers within a virtual globe such as Google Earth, Microsoft Virtual Earth, and NASA World Wind. The validity of the virtual globe representations are subject to the scrutiny of the public. Satisfaction of user needs for specific geospatial points of interest such as location of gas stations, hotels, and restaurants have popularized the use of virtual globes and led to increased numbers of layers and density of content in those layers. For the greater scientific and engineering communities, the virtual globe GIS and customized GIS systems have furthered collaboration and technical information distribution between engineering and scientific teams [1-7]. However, while Google Earth has proven to be popular for displaying geo-specific data, the API often does not provide the users required level of data interac- tion or data retrieval necessary to support real time simulation. Further, validity issues impede expansion of the use of virtual globe for engineering and scientific tasks.

Beyond the popular virtual globe applications, there are numerous examples of customized GIS systems which are used for engineering and scientific tasks by overlaying technical and scientific user specific data on geo-specific topographic databases. Hydroseek enables geo-spatial search and linkage of hydrological data from three repositories, the National Water Information System, Chesapeake Bay Information Management System, and National Atmospheric Deposition Program to a satellite image based user query interface [4]. Microsoft is developing a system called Sensor Map which is designed to interact with real-time sensors and web based information sources to provide geo-spatial mapping of user specified data [5]. Singh and Dowerah [6] use geographic information system for mapping a variety of different geology map types of mineralized zones and em- 
bedded remote sensing data to support mineralogy exploration. $\mathrm{Xu}$ and Xie [7] identify the rapid growth in embedded Geographic Information Systems for mobile, often autonomous, platform needs by exploration engineers and scientists, and propose a general system design.

The ultimate goal of this research is advancement of valid real-time display of high fidelity scientific and engineering simulations from mobile platform as layers in virtual globes. The specific topic of this research involves real-time display of dynamically varying simulations of electro-magnetic fields occurring on celestial bodies simultaneously in both mobile platforms and virtual globe systems.

\section{Case Study Architecture Requirements}

Our specific case study focuses on the software architecture needed for dynamic and simultaneous display on mobile platforms and in a virtual globe GIS of valid real-time simulations of communication coverage maps for missions on a lunar analog site. Valid simulated coverage maps portend to advance the safety of manned and robotic exploration teams through increased assurance of communications between explorations teams remotely separated from secure base stations and supporting installations. Space exploration risks are multi-faceted both in terms of human lives and equipment [8]. Approaches to reducing these risks include mission planning, mission rehearsal, mission mirroring, after-action review, and life-cycle engineering of systems suited to meet mission requirements identified through validated, high-fidelity simulations and physical testing $[9,10]$. Display of mission data through virtual globes extends analysis and feedback for the phases beyond the confines of the mission team to interested parties throughout the world.

To advance these concepts we designed architecture, built a software prototype, conducted a field study and analyzed the results. Analysis assessed the performance and validity of simulated communication coverage maps generated by that prototype for missions on an analog lunar study site. Critical to dynamic generation is validity of the simulation communications coverage map given radio parameters, dynamic mobile relay antenna placements, surface topology, and regolith characteristics affecting radio frequency (RF) propagation and attenuation. Generation of valid simulations of dynamic communication coverage maps increases assurance that the routes taken and placements of relay antennas by mobile manned or robotic entities will maintain continuous contact with other mobile entities, fixed site base stations, and/or Earth linked communications satellites.

Desired functionality of the enabling architecture in- cludes:

- Real-time generation of valid simulated communications coverage maps for mission planning, mission rehearsal, mission mirroring, after-action review, and life cycle engineering through simulation-based acquisition;

- The capability to model real-time deployment of relay antennas to extend fixed antenna ranges and simulate valid communicate coverage maps for real-time mission re-planning opportunities and urgencies;

- Output of map data in a Google Earth viewable format to facilitate extended (global and/or celestial) mission team interaction and global feedback

Given the risks associated with lost communication between a remote exploration team and supporting teams, factors that degrade communications assurance must be understood in order to create a computer model validated for simulation of the phenomenon for the region of interest. While the scope of the factors impacting communication assurance far exceed the scope of this research, line of sight between receiver and sender represents one fundamental factor given the modeled UHF frequencies of interest in our case study. Fidelity of elevation posts within a computer model of a geo-specific region of the earth or celestial body directly impact correlation between the synthetic representation of the region within the simulation and actual region on the earth or on the celestial body. The level of elevation post fidelity and RF model needed to provide a level of assure of a valid simulation of communication coverage is partially addressed in this research.

$\mathrm{Xu}$ and Xie [7] identify basic performance criteria for mobile terminals when they state: "It's necessary to analyze smart mobile terminals such as, PDA or PMP and design a hierarchical sub-blocks vector data model. The key of spatial data organization is data index and query. The performance of spatial index will directly affect the whole system performance." These performance requirements were similar to our identified basic requirements. In addition to the assumed need for a high performance spatial index and query for UHF frequency pairings is correlation of UHF as well as other expected future electro-magnetic pairings with virtual globe GIS layers. Hence a layered software architecture was envisioned to provide rapid and high-fidelity, geo-specific layers of information that could correspond to virtual globe GIS layers. Layers within a user-manipulated engineering or scientific database also enables the use of algorithms (such as line of sight calculations) to be performed on the specific data types within that layer in order to create corresponding additional layers of information. 
To shorten development time, existing layered software architectures were considered for modification and reuse. Primary considerations were given to high resolution representation of terrain, rapid calculation of line of sight, and operation on mobile autonomous platforms. One layered architecture meeting these criteria and identified for possible use currently supports training. This was also consistent with the mission related goals of our case study. The fundamental capabilities of the layered architecture identified derived from its training mission include:

- A terrain grid resolution of 1 meter.

- $10 \mathrm{~cm}$ elevation accuracy.

- Optimized Line-of-Site (LOS) calculation.

- Capability to run on a man-portable computing device (Palm PC).

In mission rehearsal, for example, terrain knowledge from a very accurate layered architecture database is used in the line-of-sight calculations to prevent modeled projections from passing through hills or other represented features. Further the layered architecture identified had a proven record of rapid, near real-time line of sight (LOS) calculation on terrain with one meter post separation [11-13]. However, past implementations of this layered architecture typically only encompassed a 2 $\mathrm{km} \times 2 \mathrm{~km}$ physical area. This physical area was far less than our expected physical area thus creating a research question, what level of computational performance will be demonstrated on a far greater physical area?

The layered architecture also leveraged commercial graphics and computer science domains to implement data storage structures and algorithms which provide optimized LOS calculation. Terrain data is stored in pages that represent 1 square kilometer of terrain. Terrain elevations are provided as a 16 bit integer value at grid post spacing of 1 meter. Culling grids are defined for 10 by 10 post areas and for larger 100 by 100 post areas. The terrain skin is stored in a hierarchical three level tree structure - the lowest level being the 1 meter grid and the highest being the $100 \times 100$ meter post grid.

To calculate line of sight the layered architecture uses a two-dimensional digital difference analyzer, which is highly optimized to rapidly traverse a regularly spaced grid. The LOS routine checks the ray against height of terrain within culling grids which it crosses, starting with the largest grid and calculating child nodes (smaller grids) only if the ray is lower than the terrain height of the parent grid.

Features in the layered architecture are represented as leaf nodes in a bounding volume hierarchy $(\mathrm{BVH})$ tree. Each node in a BVH tree is a spatial volume that fully contains all of it child nodes. Geometry of individual nodes can be basic or complex geometry types: ellipsoids, columns, triangle meshes, etc. Higher level culling volumes use simple solid geometry types to allow faster intersection checks. The feature intersection algorithm detects root node intersections and if the intersected node is defined as a culling node then its child nodes will be checked for intersection. The BVH tree is optimized for attenuated LOS calculations and will always check closest nodes first. Material attributes can be assigned to features in order to calculate attenuation of the LOS ray.

For this particular architecture, the terrain database representation and runtime reasoning services operate entirely in Cartesian coordinates, with no assumptions whatsoever about the planetary reference ellipsoid. All calculations are performed in local Cartesian coordinate systems tangent to the reference ellipsoid; a global Cartesian system is used to coordinate between different local systems. Due to the Cartesian representation, layering architecture has no difficulty handling high-latitude or polar regions.

\section{Case Study Methodology}

To address the requirements of this GIS case study, improvements to above layered architecture needed to be performed. First, a simulated communications coverage layer needed to be created within the layered architecture that included the capability to input radio antenna locations, and radio transmission properties, antenna gain, and antenna height. This data was sufficient to support a simple communications propagation and attenuation algorithm, the Friis transmission equation, which calculates signal strength at the receiving antenna [14]. LOS needed for RF communications was implemented as a layer in the architecture using Global Position System (GPS) locations and sensor direction to calculate the signal trajectory. The resulting layer of signal trajectories were viewed as communication coverage maps through the existing interface that was modified to accommodated visualization of the added communications layer components. Controls added to the interface included antenna placement, antenna parameter and transmission power specification, and selection of RF model.

In addition to the performance features of a successful GIS identified above, must be added analysis of the underlying validity of the outcomes of the information search results. UHF signals can be blocked by intervening terrain. To examine the impact of elevation post fidelity on simulated UHF communication coverage map validity, two layered architecture databases for the analog lunar region were created using different resolution source data [15]. The lower resolution elevation post database considered was the Shuttle Radar Topography (SRTM) Digital Terrain Elevation Data (DTED) Level 2 
data with approximately 30 meter elevation post separation. The higher-resolution elevation post database considered was the Light Detection and Ranging (LIDAR) database with one meter post separation. Both databases were obtained for a $27 \mathrm{~km} \times 27 \mathrm{~km}$ lunar analog site that included areas of interest for our case study. While SRTM database post separation met DTED Level 2 standards, the accuracy of elevation posts within far exceeded the minimum DTED accuracy standards. For the purposes of creating the communication coverage map within our layered architecture, the SRTM database was interpolated to within 10 meters. Due to computation load issues within the notion of at maximum an overnight mission planning scenario, the LIDAR database post separation was increased by sampling every 10 meters posts from the original database. The fidelity of the terrain representation is of interest as greater density, higher accuracy elevation posts are expected to yield a more valid simulated communication coverage map but are more costly to create and computationally demanding to use. A communication coverage map using the SRTM database for this region took approximately 2 hours to create. A communication coverage map using the 10 meter sampled LIDAR database for this region took approximately 12 hours to create (suitable for an overnight mission planning scenario).

Figure 1 below graphically depicts the resulting lay- ered architecture used in the case study.

Figure 2 below graphically depicts the entity relationships within the layered terrain:

The combination of signal propagation and attenuation with elevation postings created the basic components to produce and demonstrate a simulated mission-time communications coverage map in the synthetic representation of the region of interest. To enhance readability by the user, the simulated communications coverage maps used selectable coloration to indicate signal strength at each location. Color indication of signal strength aids ease of use and rapid comprehension of the coverage map. Three color levels (shown in Figures 3 and 4) represent signal strength used; green represents $802.11 \mathrm{G}$ capable data rates, yellow indicates $802.11 \mathrm{~A} / \mathrm{B}$ capable data rates and no color indicates lower than $802.11 \mathrm{~A} / \mathrm{B}$ or no communications signal. Figure 3 provides a screenshot showing a coverage map rendered in the layered architecture viewer.

The capability to export coverage maps to Google Earth compatible kml format was also created. Figure 4 provides a screenshot of an exported $\mathrm{kml}$ format communication coverage map as viewed in Google Earth.

The above engineering research created and demonstrated a user-friendly, layered architecture for a mobile platform for two different kinds of simulated communication coverage maps (LIDAR and STRM). The cover-

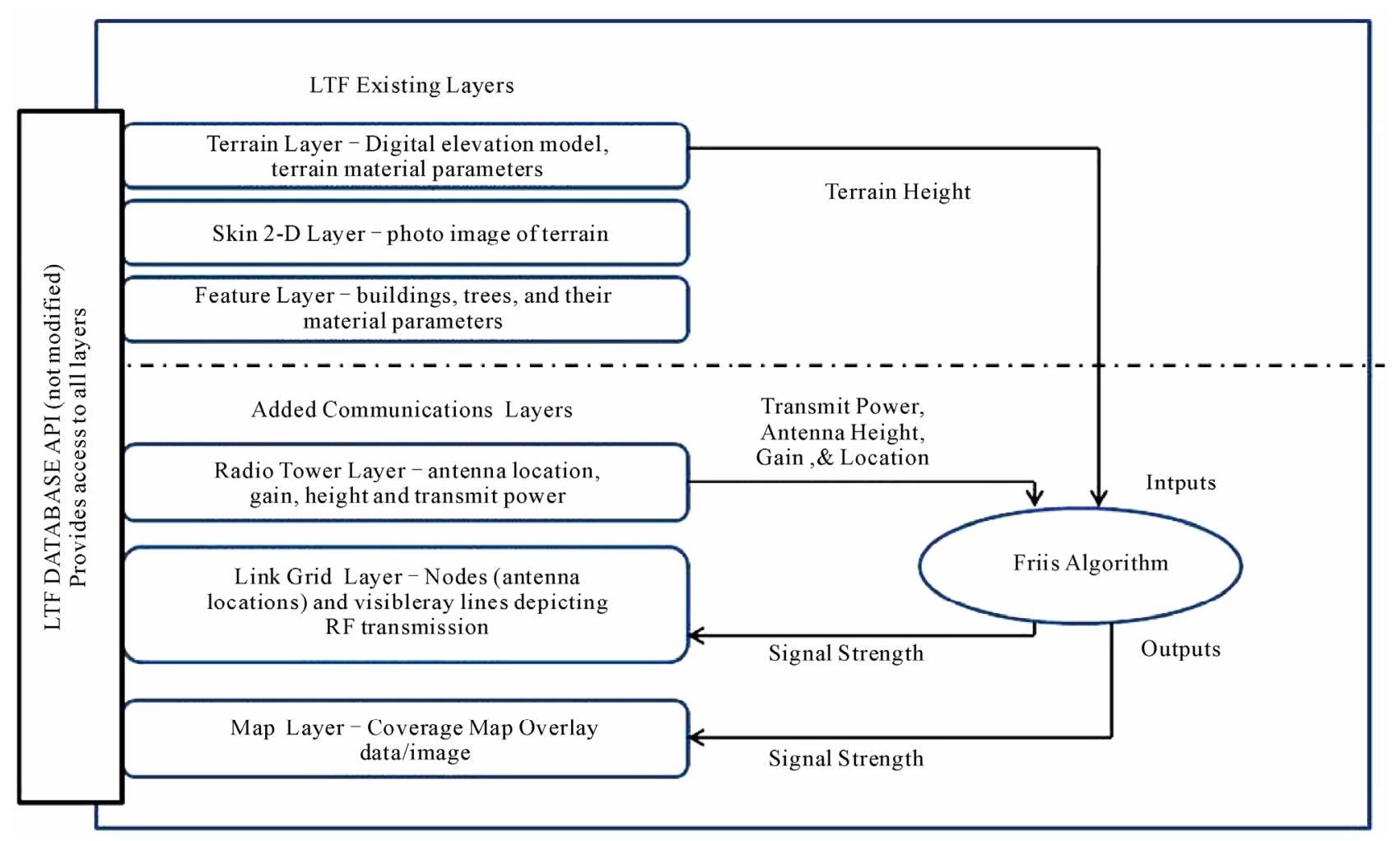

Figure 1. Layered architecture with communication layer added. 


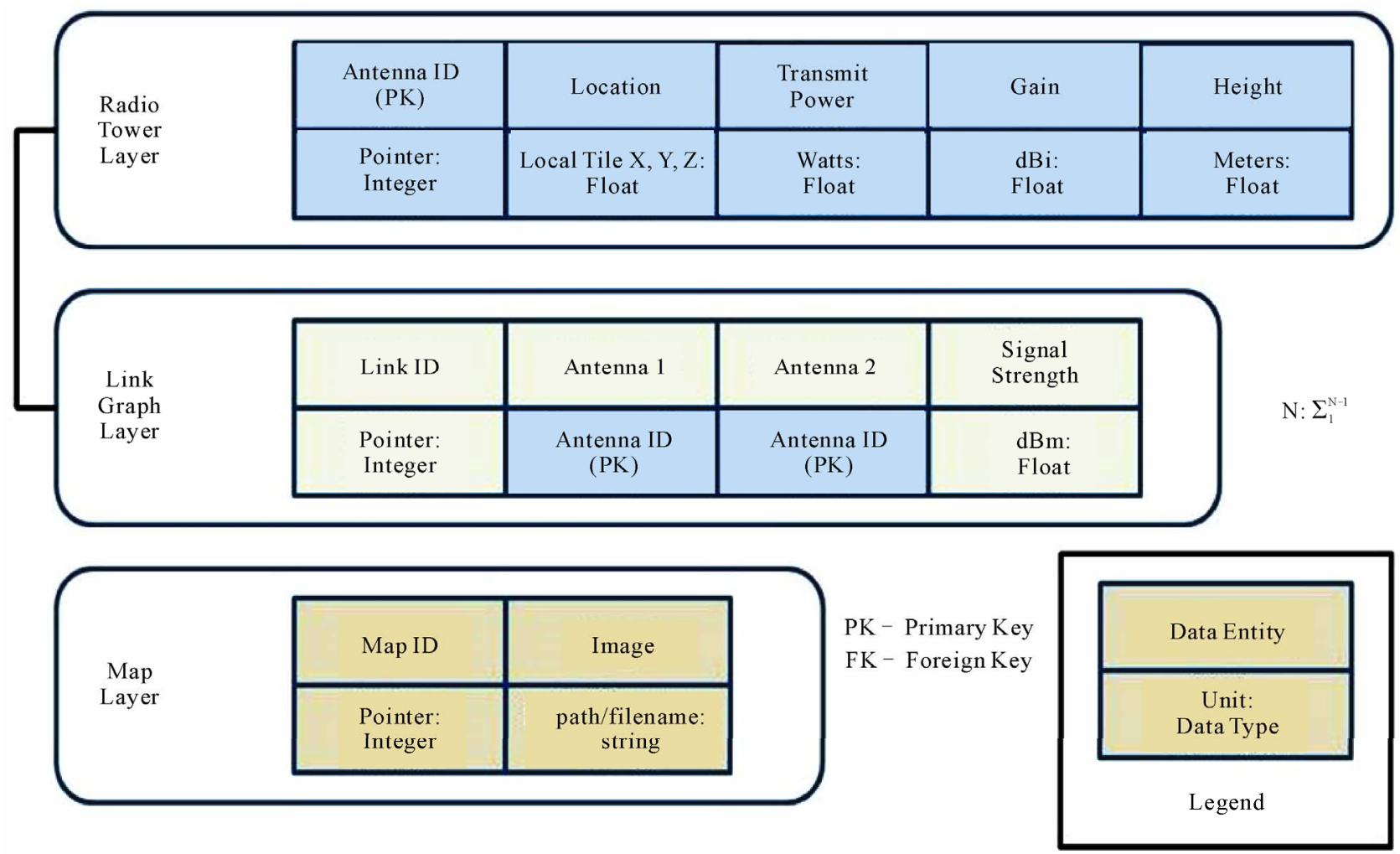

Figure 2. LTF communication layers entity relationship diagram.

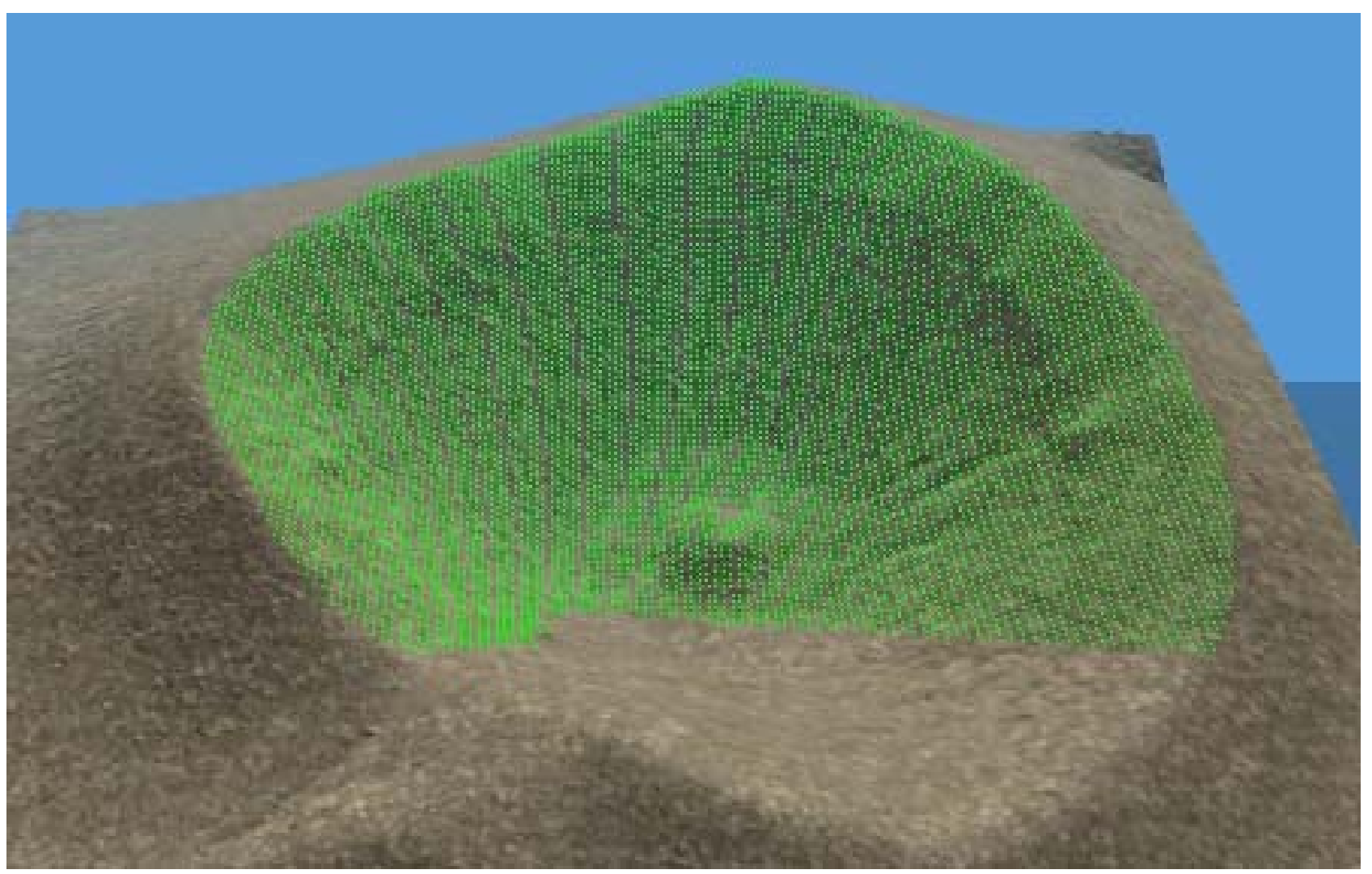

Figure 3. Simulated communication coverage map as shown in the mobile platform viewer. 


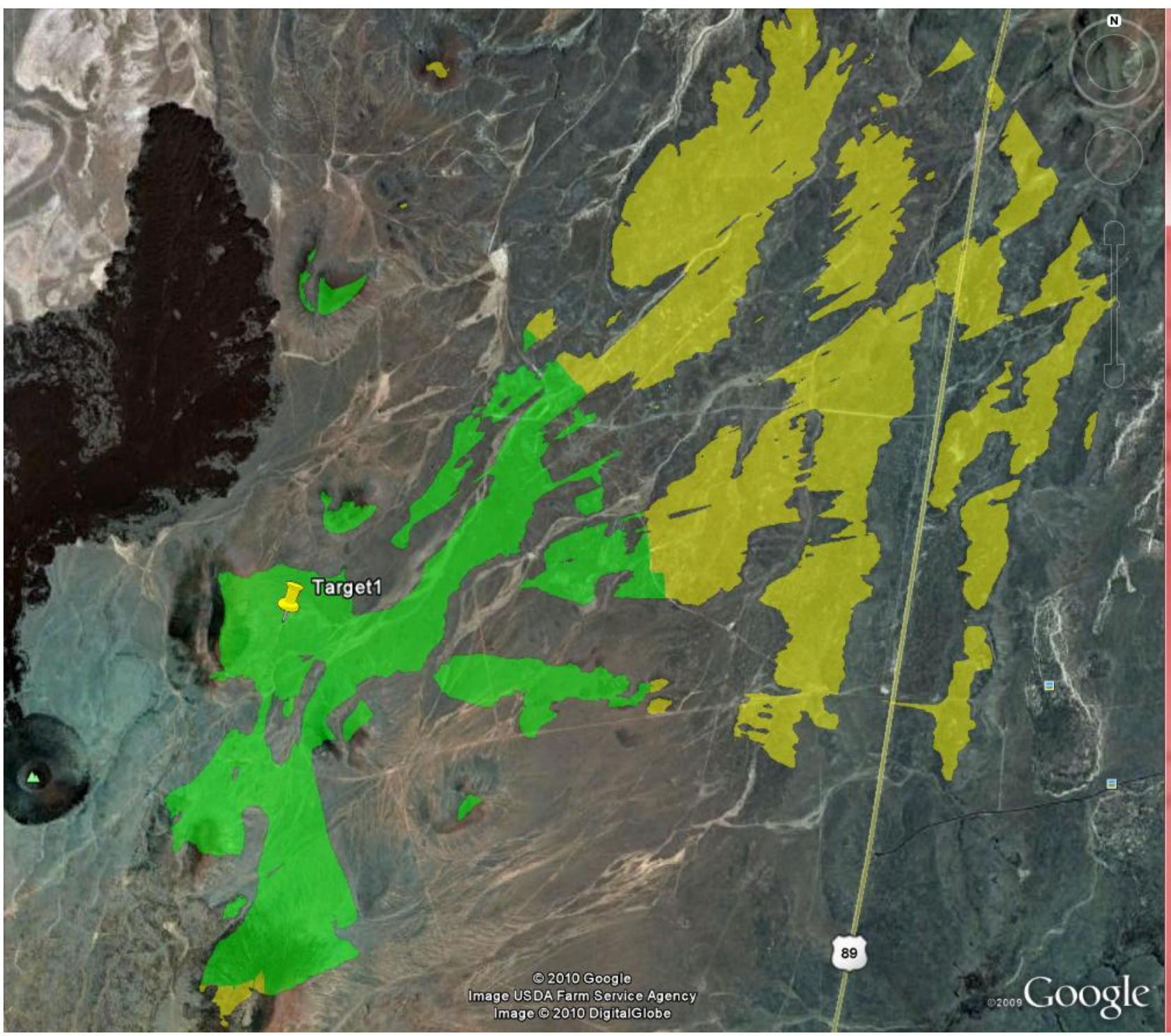

Figure 4. Simulated communication coverage map depicted in Google earth.

age maps could be displayed simultaneously on both the mobile team handheld device and within a virtual globe. The level of validity of the simulation of the two different map kinds was still unknown.S

\section{Case Study Results}

For validation purposes, the research team conducted a two day field study collecting RF transmission signal strength data throughout the identified area. Three sets of data were collected each using one fixed radio/antenna and one mobile radio/antenna. The radios used were Tropos Network Routers. The Tropos radios support 2.4 Ghz transmissions which are one of the frequencies specified in the NASA Lunar Communications Architecture [16]. The Tropos radios also provide a data port from which transmission and reception parameters could be easily recorded. GPS location data of each antenna was also recorded for each transmission event.

Antidotal observations during the field study indicated that the pre-generated coverage maps were successful in determining at what points on a route communications would or would not be available as well as determining placement of relay antennas to extend communications coverage areas.

Subsequent to the field study, statistical analysis was conducted comparing the collected RF data with the simulated RF coverage maps in order to consider the validity of the simulated coverage maps given the fidelity of the terrain databases and the signal propagation and attenuation model used. Some of the results of this analysis include: 
- Hypothesized homogeneity between the accuracy of the simulated go/no go communications coverage maps generated using LIDAR and SRTM terrain databases respectively for within range $R F$ signals could not be rejected based on sampled observed field data $\left(n=376, \chi^{2}=1.211 ; p=0.2711\right.$; Yates' $\chi^{2}=0.957 ; p=0.3279$; Post hoc analysis: Input: $\alpha=.05, \hat{w}=.3$, Output: $\beta=0.017$ )

- Observed reliability of the sampled within signal range simulated go/no go communications signal was found to be:

○ Reliability $($ LIDAR $)=0.793$

O Reliability $(S R T M)=0.745$

- Using the LIDAR layered architecture database, the hypothesized equivalence of the Friis $\mathrm{S}$ band model predict individual unblocked signal strength with observed signal strengths during the field study could not be rejected ( $n=64$; critical $t=3.32$ observed $t=1.714 ; p=0.0915$ ). However, due to the closeness of the $p$ value to 0.05 , linear regression of the predicted signal strength error (predicted signal strength - measured signal strength) plotted against distance of transmission was performed. This analysis revealed a least square line of $\mathrm{Y}=-14.049+0.004399 \mathrm{X}$. The coefficient of determination $(r 2=0.476)$ indicates that approximately $50 \%$ of the signal strength error may be attributable to distance. The remaining signal strength error may be from other sources such as signal reflection, refraction, and diffraction that are not accounted for by the Friis equation or by errors in antenna gain or transmit power values which were are modeled in the Friis equation but were assumed constant throughout the experiments.

\section{Conclusions}

The greater engineering and scientific community are increasing their attention on the possible use of virtual globe GIS to rapidly distribute real-time technical information not only to direct research and engineer team members but within larger global communities. Critical to the success of information from embedded to virtual globe GIS are performance features identified by $\mathrm{Xu}$ and Xie. Additionally, acceptance by the engineering and the scientific communities of geographic information systems as forums for information dissemination relies on confidence in the validity of the information and its' accurate association with other relevant information. We have identified that multi-purposed software like layered architectures further facilitate the dissemination of technical and scientific information from mobile platforms to virtual globe GIS. Further, interfaces that ease manipula- tion of information layers further enable operational realtime capability. Lastly communication to the global community of the level of validity of the displayed real-time simulation remains a concern as false negatives and false positives and data misinterpretation may lead to adverse decisions and outcomes.

For demonstration and validation purposes, this case study successfully implemented a layered architecture for a celestial body analog site and created communications layers within a layered GIS architecture that provided simulated RF communications coverage maps of a known level of validity. Given the selected RF model that was implemented, our results found that for the levels of assurance associated with simulated communications coverage maps generated were equally acceptable. For our level of validity, the publically available DTED Level 2 SRTM data was as valid as the coverage map generated from the more costly higher resolution database. The highest level of resolution of elevation post mapping was not tested due to required computational time beyond acceptable mission planning windows. This would suggest that careful analysis of not only execution requirements (time, computation footprint) as inferred by $\mathrm{Xu}$ and $\mathrm{Xie}$, but the impact of the algorithms operating on the stored data and the fidelity of that data should be performed in light of validity requirements. To facilitate use by global participants through a virtual globe, accuracy and/or validity levels of the scientific and engineering information being output appears to be a necessary to impart appropriate levels of assurance about the layer of information so as to avoid misinterpretation.

\section{Future Research}

Layered architectures in mobile platforms that contain validated data correlated with virtual globe layers are ripe for future investigation and research. Obvious areas for future research with respect to our case study would involve reducing the distance and electro-magnetic model related error cited above. If sufficient computational power is available to generate the coverage map within the specified mission scenario time windows, another future research area would be use of one meter terrain database for LOS calculation also cited above.

More general additional areas for future investigation include:

Non-Earth Models: Research is needed on the greater curvature of the Moon or other celestial body vs. the Earth surface to determine the optimal terrain tile size. Each flat earth terrain tile $(1 \mathrm{~km} \times 1 \mathrm{~km})$ is normal to the systems local $\mathrm{Z}$ vector introduces a minimal error (within the $10 \mathrm{~cm}$ accuracy requirements) at the edges of the tile. Reducing terrain tile sizes will minimize errors at tile 
edges but increase processing time. Elongated ellipsoids combined with this Cartesian coordinate approach may also permit the modeling of non-spherical bodies such as near earth objects.

Subsurface and above surface Models: Research is needed on determining what subsurface or above surface geo-specific data to represent, the nature of the representation, and the level of fidelity is needed. For instance mineralogy could be defined in a subsurface layer by defining 3 dimensional geometric polygonal shapes containing mineral properties. Similar polygonal shapes defined above the terrain surface could be used to represent location specific data of interest to the user such as magnetic or radiation fields.

Navigation Simulation: Research is needed on the contribution of wirelessly streamed terrain database updates augment or improve pre-mission generated terrain databases with locally collected terrain data.

Heliophysics layers and Route Planning: Research is needed on the degree and nature of layered representations of heliophysical exposure elements are appropriate. Heliophysical exposure increases danger to crews but conversely may support energy recharging requirements. Heliophysical effects layers may enable route planning in areas shadowed by mountains or craters, or maximizes solar exposure in order to keep power array output at its peak may be appropriate.

\section{References}

[1] A. J. Chen, G. Leptoukhm, S. Kempler and L. P. Di, "Visualization of NASA Earth Science Data in Google Earth," Geoinformatics, Proceedings of the SPIE, Vol. 7143, 2008, pp. 29-42.

[2] R. Kamadjeu, "Tracking the Polio Virus down the Congo River: A Case Study on the Use of Google Earth in Public Health Planning and Mapping," International Journal of Health Graphics, Vol. 8, No. 4, 2009, pp. 1-12.

[3] M. N. K. Boulos, M. Scotch, K.-H. Cheung and D. Burden, "Web GIS in Practice VI: A Demo Playlist of Geo-Mashups for Public Health Neogrographers," International Journal of Health Geo- graphics, Vol. 7, No. 38, 2008, pp. 1-16.

[4] B. Beran and M. Piasecki, "Engineering New Paths to Water Data," Computers \& Geosciences, Vol. 35, No. 4, 2009, pp. 753-760. doi:10.1016/j.cageo.2008.02.017
[5] S. Nath, J. Liu and F. Zhao, "SensorMap for Wide-Area Sensor Webs," IEEE Computer, Vol. 40, No. 7, 2007, pp. 90-93.

[6] B. Singh and J. Dowerah, "Geospatial Mapping of Singhbhum Shear Zone (SSZ) with Respect to Mineral Prospecting," Journal of Geographic Information system, Vol. 2, 2010, pp. 177-184. doi:10.4236/jgis.2010.23025

[7] Z. Y. Xu and Z. Xie, "Research on Key Technology of General Embedded GIS," Journal of Geographic Information System, Vol. 2, 2010, pp. 15-18. doi:10.4336/igis.2010.21004

[8] NASA/SP-2004-6113, "Bioastronautics Roadmap, a Risk Reduction Strategy for Human Space Exploration," NASA Scientific and Information Program Office, February 2005.

[9] J. F. Connolly, "Constellation Program Overview," NASA Presentation, 2006.

http://www.nasa.gov/pdf/163092main_constelltion_progr am_overview.pdf

[10] D. Monell, "NASA Constellation Program Modeling and Simulation," NASA Presentation, May 2007.

[11] S. Borkman, G. Peele and C. Cambell, "An Optimized Synthetic Environment Representation Developed for OneTESS Live Training," Interservice/Industry Training, Simulation, and Education Conference, 2007.

[12] J. Campos, S. Borkman, G. Peele and C. Cambell, "Toward Cross Domain Terrain Services," Interservice/Industry Training, Simulation, and Education Conference, 2008.

[13] W. Baer, T. R. Campbell, J. Campos and W. Powell, "Modeling Terrain for Geo-pairing and Casualty Assessment in OneTESS," Modelling and Simulation for Military Operations III, Proceedings of SPIE, Vol. 6965, 11 April 2008.

[14] J. Lavergnat and M. Sylvain, "Radio Wave Propa- gation Principles and Techniques, " John Wiley \& Sons, Ltd., West Sussex, 2000.

[15] NASA Website, "NASA's Desert Research and Technology Studies (D-RATS)," 2010. http://science.ksc.nasa.gov/ d-rats

[16] J. Schier, "NASA's Lunar Space Communication and Navigation Architecture," American Institute of Aeronautics and Astronautics, September 24, 2007.

[17] B. McLarnon, "VHF/UHF/Microwave Radio Pro-pagation: A Primer for Digital Experimenters," TAPR/ARRL Digital Communications Conference, 1997. http://www.tapr.org/ve3jf.dcc97.html 